\title{
EFECTOS EN LA SUBJETIVACIÓN DE ADOLESCENTES EN CONFLICTO CON LA LEY PENAL QUE PARTICIPAN EN PROGRAMAS DE PREVENCIÓN SECUNDARIA Y TERCIARIA
}

Silvana Contino

Magíster en Psicología de la UDELAR Profesora adjunta del IUPA y de la UDELAR Miembro de la AUDEPP

Correo electrónico: silvana.contino@gmail.com ORCID: 0000-0002-0978-9170 


\section{Resumen}

A partir del trabajo profesional de coordinación territorial de equipos técnicos de programas que abordan situaciones de adolescentes en conflicto con la ley penal, y de la investigación y formación académica en el tema de adolescentes que incurren en conductas delictivas, se propone como objetivo problematizar las implicancias en los procesos de subjetivación adolescente de los que participan en los programas que responden a la prevención secundaria y terciaria en la violencia juvenil. Programas diseñados con tal objetivo dejan, en ocasiones, atrapado al adolescente en una lógica en la cual las propias políticas públicas solamente pretenden la inclusión social y la no vulneración de derechos. El diseño de estos programas pone el acento en una protocolización de las situaciones abordadas, en vez de atender la particularidad o singularidad subjetiva del adolescente. La normatización de las situaciones en una lógica de derechos genera violencia en los beneficiarios del programa, por lo cual quedan desdibujados los tiempos lógicos internos de cada sujeto, su familia y referencias adultas de aquellos adolescentes que son población objetivo.

Palabras clave: subjetivación adolescente, instituciones, prevención, adolescentes en conflicto con la ley

\section{THE EFFECTS OF SUBJECTIVATION IN ADOLESCENTS IN CONFLICT WITH THE LAW WHO PARTICIPATE IN SECONDARY AND TERTIARY PREVENTION PROGRAMS}

\section{Abstract}

Emerging from the professional territorial coordination work of technical teams of Programs that address situations with adolescents in conflict with the law, research and academic training on the subject of adolescents who engage in criminal conduct, our objective is to problematize the implications in the processes of adolescent subjectivation of those who participate in programs that respond to secondary and tertiary prevention in youth violence. Programs designed for this purpose sometimes leave the adolescent trapped in a logic from which public policies only try to reduce social exclusion and violation of their rights. The design of these programs puts emphasis on a protocolization of the situations addressed, instead of addressing the adolescent's particularity or subjective singularity. The standardization of these situations, in a logic of rights, generates violence in the beneficiaries of the program, blurring the lines of the internal logical times of each subject, their family and adult referents of the adolescents in the target population.

Keywords: adolescent subjectivity, institutions, prevention, adolescents in conflict with the law 


\section{INTRODUCCIÓN}

Al pensar sobre las condiciones de reclusión de adolescentes en conflicto con la ley y sus procesos de subjetivación, entendía que las condiciones actuales de reclusión se le imponen al sujeto que delinquió desde la lógica de la fuerza, de lo intrusivo; son efecto del poder y lo instituido es, consecuentemente, de carácter violento. Ello tiene repercusiones en la subjetividad de las personas que se encuentran en tal condición. Si bien este tema es interesante para su discusión, me preguntaba también qué pasaba con las situaciones en las que no se llega directamente a la medida de reclusión y los sujetos que cometen delitos participan en ciertos dispositivos pensados como alternativas a la reclusión. Se trata, en definitiva, de programas cuyo carácter es procurar la prevención de las conductas delictivas y la disminución de la reincidencia. Son programas que responden a políticas públicas sobre el tema, diseñados por organismos del Estado y que están, básicamente en su mayoría, orientados a una población específica, como es el caso de los adolescentes en conflicto con la ley penal. Por lo tanto, decidí plantear y problematizar este tema a partir de mi trabajo profesional, como investigadora y académica en el tema de adolescentes que incurren en delitos, e indagar los efectos en el proceso de subjetivación adolescente en tanto se participa en dispositivos de prevención secundaria y terciaria de la violencia juvenil.

En los últimos años, los adolescentes que incursionan en conductas delictivas han cobrado relevancia en la opinión pública. La difusión en medios de comunicación y en las redes sociales lo configura como tema de preocupación social, de salud y jurídico-legal. Sin embargo, estudios 
coinciden en que el número de adolescentes que cometen delitos son significativamente menor en comparación con las actividades delictivas de los adultos (López y Palummo, 2013). En el año 2014, se suscitó una discusión en torno a una propuesta de reforma constitucional que pretendía, con diferentes argumentos, bajar la edad de imputabilidad para los adolescentes que cometieran delitos en el país. La sociedad en general debatía si los jóvenes debían ser juzgados como adultos a los 16 o a los 18 años de edad. Se consideraba que el sujeto puede ser penalmente responsable dada su capacidad de actuar motivado por las normas, «salvo que tenga algún defecto o alteración psíquica que incida en su imputabilidad» (Muñoz Conde, 1985, en Barquet et al., 2014).

En esta coyuntura y dados los insuficientes votos en el plebiscito que tuviera lugar para bajar la edad de imputabilidad, comienzan el diseño y la implementación de programas que involucran a varios organismos e instituciones estatales y que tienen como objetivo la prevención de conductas delictivas y la disminución de las reincidencias en adolescentes entre 13 y 17 años de edad que se encontraban en conflicto con la ley.

En general, la población beneficiaria de estos programas son adolescentes que han incursionado en conductas delictivas y las bases epistemológicas que sustentan dichos programas se encuentran en la lógica de vulneración de derechos. Por lo regular, en el diseño de los instrumentos aplicados se apunta a jóvenes que provienen de barrios catalogados como marginales y de familias vulnerables. En el protocolo de intervención participan diversas instituciones, cada una con sus lógicas, para restituir aquellos derechos que fueran vulnerados y dar así cumplimiento al objetivo del programa. El supuesto básico es que ciertos derechos vulnerados propician la comisión de delitos en los jóvenes. Si bien las instituciones se encuentran disponibles para ponerse en marcha en un trabajo de red intersectorial, en el trabajo territorial y de coordinación queda en evidencia que, a partir de sus lógicas institucionales y sus distintas funciones en 
el Estado, se desprenden concepciones de una adolescencia minorizada, lo que obstaculiza abordajes personalizados y singularizantes. Se deja por fuera la posibilidad de pensar las particularidades de las subjetividades adolescentes en juego, como procesos de construcción de subjetivación adolescente.

Como profesional, académica e investigadora, considero que acciones de políticas públicas sobre la adolescencia minorizada, ancladas en la perspectiva de derechos de infancia solamente, tienden a protocolizar ciertas acciones, lo cual deriva en una suerte de cristalización de las intervenciones. Esto se expresa en cierta incapacidad para percibir y albergar la singularidad de los adolescentes, así como de sus tiempos subjetivos. Se repite así la violentación al sujeto a partir del reforzamiento de prácticas de control y tutela, lo que los ubica nuevamente en el lugar de la carencia.

\section{ALGUNAS CONCEPTUALIZACIONES}

Es posible definir la subjetividad como un concepto que tiene que ver más con lo sociológico que con lo psicoanalítico propiamente dicho, más allá de que en muchas ocasiones se haga uso de su definición en la práctica (Bleichmar, 2003). La subjetividad, como configuración subjetiva (González Rey, 2010), entrama las condiciones materiales y simbólicas de existencia, y las instituciones sociales matrizan el proceso de socialización del sujeto y revelan el modo singular de estar en el mundo con otros. Esta singularidad contiene a su vez las dimensiones cognitivas, emocionales-afectivas y de las prácticas (González Rey, 2010; Bonvillani, 2009; Bleichmar, 2003). La producción de subjetividad hace al modo en que las sociedades determinan las formas en las que se constituyen sujetos que puedan integrarse a sistemas que les otorguen un determinado lugar. La 
subjetividad hace a un conjunto de elementos que configuran un sujeto fuertemente marcado por lo histórico y por las representaciones con las cuales cada sociedad determina aquello que considera necesario para la conformación de los sujetos aptos para desplegarse en su interior. Por otra parte, la subjetividad varía de acuerdo a cada momento histórico y se transforma análogamente junto a los sistemas histórico-políticos. Las condiciones históricas, políticas, económicas, socioculturales, familiares, transubjetivas e intersubjetivas dan material representacional para lo intrasubjetivo y para la construcción del psiquismo (Bleichmar, 2003).

El proceso de subjetivación adolescente no escapa de esta definición e implica la puesta en juego de una multiplicidad de factores que interaccionan. Marcelo Viñar (2009) plantea que el proceso de subjetivación adolescente es un proceso de transformación complejo y difícil, en el que lo biológico, lo psicosocial, lo histórico, lo sociocultural, lo económico y lo político se ponen en juego. Por ello habla de adolescencias y no de adolescencia como categoría preestablecida. Durante la modernidad era posible escuchar «los jóvenes son el futuro» (Klein, 2008) porque imperaba la idea de dar paso a una construcción sólida que apostaba a la inversión en la juventud como impulsora de valores transmisibles y sostenibles. En la actualidad, con la hipermodernidad y la cultura de lo efímero, no perdurable, líquido, y de valores poco duraderos o superficiales por lo cambiantes, se condena al joven por no poseer lo que la propia sociedad no le da. Se es injusto al condenar a los adolescentes por aquello que el mundo adulto no le ha proporcionado. Por lo tanto, al decir de Néstor García Canclini (2004), los «jóvenes no son el futuro, sino el presente de la sociedad» y somos responsables de dejarlos en una suerte de vacíos sólidos identificatorios, en tanto referentes, que deberían oficiar de soporte para el tan trabajoso costo psíquico de construcción de identidad (Di Segni Obiols, 2002; Cao, 1997, 2009, 2013; Fandiño Parra, 2011). La condición adolescente en la constitución subjetiva tiene una urgencia 
identificatoria (Cao, 1997, 2009) en la que necesita apuntalarse, y cuando ella no aparece en las formas de paternidad, estilos parentales o ciertos estilos de padres (Oliva Delgado et al., 2008; Kancyper, 2007), los apoyos sociales de pares cobran relevancia (Cumsille y Loreto Martínez, 1994). Las conductas de infracción dentro del proceso de subjetivación adolescente pueden ir, en el mejor de los casos, desde una lógica confrontativa (Kancyper, 1997), exploratoria de conductas de riesgo (Le Breton, 2011), como formas fallidas de encontrar la estabilidad que la condición adolescente hace tambalear (Cao, 2009), hasta la necesidad de ciertas conductas implementadas para la construcción de una rápida identidad «falsa», que sirva como soporte ante el sufrimiento por tanto vacío y desamparo (Winnicott, 1981).

Desamparo es un término pensado por el psicoanálisis. Desde el lenguaje corriente se puede definir como una situación o estado de desprotección, desvalimiento o vulnerabilidad. El desamparo da cuenta de alguien que no recibe el apoyo o la ayuda que necesita. Jean Laplanche y Jean-Bertrand Pontalis (1996) plantean que el desamparo se define como el «estado del lactante que, dependiendo totalmente de otra persona para la satisfacción de sus necesidades (sed, hambre), se halla impotente para realizar la acción específica adecuada para poner fin a esa tensión interna» (p.94). A pesar de que esta definición esté orientada al desamparo en la infancia, se puede ver en la adolescencia situaciones análogas o similares.

Contemplando la noción puramente psicoanalítica de Laplanche y Pontalis (1996), se podría afirmar que el desamparo en la adolescencia sería el estado en el que el adolescente, al depender de otros para la satisfacción de sus necesidades psíquicas de construcción de identidad, se halla impotente para realizar la acción específica adecuada para poner fin a esa tensión interna que implica la conformación sólida e integrada de las identificaciones necesarias para tal construcción, sin que quede un vacío en ello. En dicho proceso, lo fundamental es el rol que ocupan las figuras 
parentales, referentes e instituciones como soporte de la construcción subjetiva. La noción de desamparo da cuenta de que es necesario que exista otro que ampare, acompañe y apuntale (Cao, 2013). Myrta Casas de Pereda (2018) propone una definición precisa de amparo, que permite evidenciar de qué se habla cuando se habla de desamparo:

El amparo implica otro que rodea y remite a todo aquello del orden de la realidad afectiva que protege de las fuerzas exteriores, del posible daño. Y al mismo tiempo, implica en el orden de la vivencia (fantasía) la necesidad expresa de un afecto, del compromiso libidinal del otro en esa función de cuidado y protección. (p. 11)

Marcelo Luis Cao (1997) desarrolla el concepto de apuntalamiento. Lo describe como un concepto fundamental para la estructuración, formación y desarrollo del psiquismo, que opera constantemente a lo largo de la vida del sujeto de forma de que los otros, en todas sus formas, puedan acompañar el proceso de subjetivación. De acuerdo con esta perspectiva, el apuntalamiento se despliega en tres dimensiones. La primera es la del apoyo sobre una base originante, que en el caso de la adolescencia está relacionada con las primeras imágenes parentales. La segunda dimensión es la de la modelización, que consiste en el trabajo de identificación que opera sobre los otros del vínculo, que forman parte de la experiencia vivencial del sujeto. La tercera es la ruptura crítica, dimensión relacionada con las pérdidas que acarrea el proceso de maduración; esta corresponde al concepto de transcripción e implica la puesta en marcha de un trabajo elaborativo, que permite el reposicionamiento del sujeto en las dimensiones intersubjetiva e intrasubjetiva dentro del campo representacional.

Es durante la adolescencia que estas dimensiones toman especial valor y espesor, en tanto las viejas apoyaturas que servían en la niñez deben ser desechadas e intercambiadas por otras que den cuenta del proceso 
madurativo del joven. En este sentido, comienzan a tallar los apoyos sociales recibidos y percibidos de diferente orden, y no solo desde lo intersubjetivo, sino en relación con el soporte interinstitucional (Cumsille y Loreto Martínez, 1994). Ante situaciones de vacíos identificatorios sostenidos, de fallas en el apuntalamiento a nivel imaginario y simbólico en un momento primario, las instituciones referenciales pueden oficiar como aquellos apoyos faltantes y dar sostén, en una segunda oportunidad, a la construcción de subjetividad. Si las instituciones no albergan y sostiene al joven, porque se encuentran en crisis, dejan a los adolescentes a la deriva (Di Segni Obiols, 2002).

Hay desarrollos teóricos en referencia a las conductas delictivas en la adolescencia o violencia juvenil, que con sus estudios tratan de darle explicación o caracterizar ese modo de funcionamiento. Históricamente han recibido la influencia de los desarrollos criminológicos en la esfera del ámbito adulto hasta llegar, en los años 70, a desarrollos específicos en el área. Estas líneas de desarrollo, en lo que hace a la comprensión de la conducta delictiva en la adolescencia, se apoyan en diferentes paradigmas. Algunas, las más clásicas, hablan de la "carrera delictiva» en un sujeto; estas han estudiado que en el desarrollo del sujeto hay una suerte de continuidad de las conductas y que solo cambia su naturaleza e intensidad. En esta línea está la psicología de la delincuencia juvenil, la criminología del desarrollo (Redondo Illescas y Pueyo, 2007; Redondo Illescas, 2008), y hasta algunas más actuales, que hacen énfasis en la identificación de factores de riesgo y protección en el desarrollo de un sujeto en el que la presencia de dichos elementos puede o no aumentar la probabilidad de que la conducta delictiva aparezca en algún momento de su historia. Es en estos desarrollos teóricos en los que se apoyan los programas de prevención de la conducta delictiva, en tanto tienen una postura según la cual se trata de disminuir los factores de riesgo en un sujeto y su familia y de potenciar los factores de protección. Los estudios sobre factores de 
riesgo y de protección se encuentran realizados en poblaciones vulneradas en varios aspectos: a nivel familiar, socioeconómico, sociocultural, etcétera.

En ciertas poblaciones vulneradas a nivel socioeconómico y sociocultural se acentúan las carencias de soporte para la subjetivación adolescente. En estas realidades los vacíos son de carácter estructural, realidades de déficit a todo nivel, de carácter transgeneracional. No han logrado ciertas apoyaturas para los procesos de subjetivación de los sujetos que las habitan. Las diferencias socioeconómicas y socioculturales imprimen una realidad en las familias en la cual la violencia se instala como tal, a cualquier nivel de expresión, y provoca fallas y daños. Los estudios que han echado luz sobre los factores de protección y de riesgo para las conductas delictivas cometidas por adolescentes solo logran detectar una parte del problema. Dadas las falencias en las lógicas barriales, en las instituciones, en la familia y los adultos referentes, tratar que los factores de riesgo disminuyan no hace que los niños, niñas y adolescentes puedan, con la restitución de ciertos derechos, encontrar el sustento necesario para las subjetividades implicadas. En algunas oportunidades, no basta con que se coloque en instituciones que tienen esas funciones a aquellos adolescentes que quedan en los márgenes de los controles en salud, por ejemplo, o que carecen de escolarización o de inserción en la educación no formal, para que tengan contacto con otros jóvenes proactivos no delictivos, creyendo así que se los incluye y se les restablecen derechos que no estaban presentes. En muchos casos, los adolescentes «de los márgenes», por más que se les encuentre un lugar de reinserción en el sistema — sea este de salud, de educación, de formación profesional—, hacen abandono de la institución, porque las instituciones tienen una función normalizante y generalizante del deber ser, donde queda diluida la particularidad, la singularidad de cada caso, de cada familia y su historia, de la historia del adolescente y las subjetividades en cuestión. 
Entonces, la mirada y las prácticas profesionales que se orientan solo a la restitución de los derechos, que se consideran como formas de inclusión social, si no son acompañadas por una mirada y lectura constante, por un seguimiento de lo singular y de lo procesual de cada adolescente, de su subjetivación, de su dinámica familiar y de su realidad concreta social, no pueden ser sostenidas por los adolescentes. Las conductas instaladas se repiten en las instituciones implicadas y hacen que se los vuelva a desvincular, de manera que se cierra así un circuito de violentación que deja al adolescente en una suerte de repetición de la historia, en el lugar de la dureza de la violencia y de las carencias de apoyo y apuntalamiento.

\section{CONSIDERACIONES FINALES}

Por lo tanto, podemos reflexionar que las acciones de política pública sobre la adolescencia minorizada ancladas en la perspectiva de derechos de infancia tienden a protocolizar ciertos procesos, lo que deriva en una suerte de cristalización de las intervenciones posibles. Esto se expresa en cierta incapacidad para percibir y albergar la singularidad de los adolescentes implicados, así como para considerar sus tiempos subjetivos. Esto interpela, porque lo que se repite es la violentación del sujeto a partir del reforzamiento de prácticas de control y tutela, de lo que «debe ser», lo cual ubica nuevamente al adolescente y a su familia en el lugar de la carencia y ante la imposibilidad de ocupar lugares supuestamente otorgados.

A su vez, los sustentos ontológicos y metodológicos que guían estas acciones técnicas en los programas de prevención del delito en adolescentes no permiten ubicar la construcción de un dato que revele los factores de riesgo: la subjetividad de los propios técnicos y técnicas intervinientes. Aquello que sucede con los técnicos, con la tarea que realizan, 
con las historias de los adolescentes y sus familias, con el encuentro en los contextos en que habitan, se trata como un obstáculo para la realización de la tarea. En este sentido, la investigación e intervención de corte psicosocial, necesaria para abordar la temática delictiva en la adolescencia, se lleva adelante sin una concepción de las subjetividades como algo del orden de lo dialógico, de lo cual son parte las dimensiones emocionalesafectivas, cognitivas y de las prácticas de los profesionales intervinientes.

La protocolización de la intervención que llevan adelante los programas - recabar datos sobre factores de riesgo y protección y brindar orientación para el acceso a los distintos servicios en pos de la restitución de los derechos - entra en una lógica binaria entre un sujeto significado desde la carencia y una oferta de soluciones preestablecidas, de la que será acreedor. De este modo, la productividad subjetiva del encuentro entre el equipo interviniente y el adolescente y su familia no resulta sostenida por la acción pública, por lo que queda restringida la posibilidad de desarrollar aptitudes para la autogestión y la iniciativa. Los adolescentes que no se encuadran en la protocolización estipulada, que no responden a los tiempos prescritos, que cuentan con otros sufrimientos, no tienen cabida en el programa. Esto implica otras formas de violencia, con otras consecuencias en la subjetivación de los adolescentes, en una suerte de repetición, ya que no tiene cabida el ser escuchados desde el padecimiento.

Cabe preguntarse sobre los efectos de estigmatización de estas prácticas y sobre la doble inscripción del concepto de minoridad adolescente, es decir: el abandono y la infracción. Los adolescentes con bajos factores de riesgo de comisión de delitos son acogidos de forma temporal, sin que se tenga en cuenta situaciones que hacen a los momentos singulares de subjetivación, en un momento crítico como la adolescencia, y sin escuchar la particularidad y los sentidos de las conductas cometidas. Para aquellos con altos factores de riesgo de cometer acciones delictivas 
graves, multideterminadas por la extrema vulneración de los derechos sociales, las problemáticas de salud mental no tratadas, las problemáticas personales y familiares, la ausencia de figuras referentes afectivas, una lógica familiar delictiva que estaba presente como forma de identificación y fallido soporte de identidad, no se prevé acciones de apuntalamiento que permitan el sostén y el soporte subjetivo necesarios.

\section{REFERENCIAS BIBLIOGRÁFICAS}

Barquet, P., Cillero, M. y Vernazza, L. (2014). Aportes para la cobertura periodística sobre la rebaja de la edad de imputabilidad. Montevideo: Unicef Uruguay.

Bleichmar, S. (2003). La subjetividad en riesgo. Buenos Aires: Topía.

Bonvillani, A. (2009). Subjetividad politica juvenil. Estudio comparativo en jóvenes cordobeses de procedencias sociales contrastantes (tesis doctoral). Córdoba: Facultad de Psicología, Universidad Nacional de Córdoba.

CAO, M. L. (1997). Planeta adolescente. Cartografia psicoanalitica para una exploración cultural. shorturl.at/etKNW

CAO, M. L. (2009). La condición adolescente. Replanteo intersubjetivo para una psicoterapia psicoanalítica. Buenos Aires: Autopublicación.

CAO, M. L. (2013). Bordes y desbordes adolescentes. En I Coloquio internacional sobre culturas adolescentes, subjetividades, contextos y debates actuales. Argentina-Francia-Uruguay. Buenos Aires. shorturl.at/CKMW8 Casas de Pereda, M. (2018). El desamparo del desamor: a propósito de la depresión en la infancia. Revista Uruguaya de Psicoanálisis. Desamparo, 127, 11-24. shorturl.at/huOR9 
Cumsille, P. y Loreto Martínez, M. (1994). Efectos del estrés y el apoyo social sobre el bienestar psicosocial de los adolescentes: revisión de la literatura. Psykhe, 3(2), 115-123.

www.psykhe.cl/index.php/psykhe/article/view/49/49

Di Segni Obiols, S. (2002). Adultos en crisis. Jóvenes a la deriva. Buenos Aires: Novedades Educativas.

FANDIÑO PARRA, Y. J. (2011). Los jóvenes hoy: enfoques, problemáticas y retos.

Revista Iberoamericana de Educación Superior, 2(4), 150-163.

shorturl.at/cgmoy

García Canclini, N. (2004). Diferentes, desiguales y desconectados. Mapas de la interculturalidad. Barcelona: Gedisa.

GonzÁlez Rey, F. (2010). Las categorías de sentido, sentido personal y sentido subjetivo en una perspectiva histórico-cultural: un camino hacia una nueva definición de subjetividad. Universitas Psychologica, 9(1), 241-253. www.scielo.org.co/pdf/rups/v9n1/v9n1a19.pdf

KANCYPER, L. (1997). La confrontación generacional. Estudio psicoanalítico. Buenos Aires: Paidós.

Kancyper, L. (2007). Adolescencia: el fin de la ingenuidad. Buenos Aires: Lumen.

KLEIN, A. (2008). La (dramática) realidad social y psíquica de muchos jóvenes latinoamericanos. Revista Liberabit, 14(14), 21-30.

shorturl.at/ektA2

Laplanche, J. y Pontalis, J. B. (1996). Diccionario de psicoanálisis. Barcelona: Paidós.

Le Breton, D. (2011). Conductas de riesgo. De los juegos de la muerte a los juegos de vivir. Buenos Aires: Topía.

López, A. y Palummo, J. (2013). Delincuencia juvenil en la ciudad de Montevideo. shorturl.at/cyVW9

Oliva Delgado, A., Jiménez Morago, J., Parra Jiménez, A. y Sánchez QueiJa, I. (2008). Acontecimientos vitales estresantes, resiliencia y ajuste 
adolescente. Revista de Psicopatología y Psicología Clínica, 13(1), 53-

62. www.aepcp.net/rppc.php?id=185

Redondo Illescas, S. (2008). Individuos, sociedades y oportunidades en la explicación y prevención del delito: Modelo del Triple Riesgo Delictivo (TRD). Revista Española de Investigación Criminológica, 6, $1-53$.

https://reic.criminologia.net/index.php/journal/article/view/34

Redondo Illescas, S. y Pueyo, A. A. (2007). La psicología de la delincuencia. Papeles del psicólogo, 28(3), 147-156.

http://www.papelesdelpsicologo.es/pdf/1499.pdf

VIÑAR, M. (2009). Mundos adolescentes y vértigo civilizatorio. Montevideo: Trilce.

Winnicott, D. W. (1981). El proceso de maduración en el niño. Barcelona: Laia. 


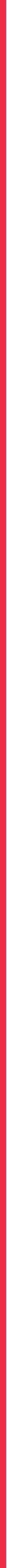

